\title{
TOTAL INDUCED VERTEX STRESS IN BARBELL-LIKE GRAPHS
}

\author{
Joseph Shiny ${ }^{1}$, Johan KoK ${ }^{2}$, V. AJitha ${ }^{3}$ \\ ${ }^{1}$ Mathematics Research Center, Mary Matha Arts and Science College, \\ Mananthavady, Kerala, India, shinyjoseph314@gmail.com \\ ${ }^{2}$ Independent Mathematics Researcher, City of Tshwane, South Africa \& \\ Visiting Faculty at CHRIST (Deemed to be a University), Bangalore, India, \\ jacotype@gmail.com \\ ${ }^{3}$ Department of Mathematics, Mahatma Gandhi College, Iritty, Kerala, \\ India, avmgc10166@yahoo.com
}

\begin{abstract}
This paper introduces new parameters called induced vertex stress and total induced vertex stress in $G$, respectively. For graphs $G$ and $H$, aspects of the maximum and minimum total induced vertex stress that can be obtained by 1-edge addition and 2-vertex merging are discussed.
\end{abstract}

Key words and Phrases: Induced vertex stress, total induced vertex stress, barbelllike graphs.

\section{INTRODUCTION}

It is assumed that the reader is familiar with the general notation and concepts in graphs. See $[1,2,6]$. Only finite, connected and undirected simple graphs will be considered throughout this paper.

In 1953, the researcher Alfonso Shimbel [4] introduced the notion of vertex stress in a graph $G$ denoted by $\mathcal{S}_{G}(v), v \in V(G)$ (see [4]). Recall that the vertex stress of vertex $v \in V(G)$ is the number of times $v$ is contained as an internal vertex in all the shortest paths between all pairs of distinct vertices in $V(G) \backslash v$. Formally stated, $\mathcal{S}_{G}(v)=\sum_{u \neq w \neq v \neq u} \sigma(v)$ where $\sigma(v)$ is the number of shortest paths between vertices $u, w$ with $v$ an internal vertex. The total vertex stress of $G$ is given by $\mathcal{S}(G)=\sum_{v \in V(G)} \mathcal{S}_{G}(v)$. For graph $G$ of order $n$, the average vertex stress of $G$ denoted by $\overline{\mathcal{S}}(G)$ follows naturally as $\overline{\mathcal{S}}(G)=\frac{1}{n} \sum_{v \in V(G)} \mathcal{S}_{G}(v)$. At present the authors

2020 Mathematics Subject Classification: 05C07, $05 \mathrm{C} 12$.

Received: 22-10-2020, accepted: 23-05-2021. 
are only aware of two papers in which the notion of vertex stress has been furthered. See $[3,5]$.

In [3] the notion of the induced vertex stress by $v_{1}$ on each of $v_{i}, i=2,3,4, \ldots, v_{n}$ on a path $v_{1} v_{2} v_{3} \cdots v_{n}$ was introduced. The induced vertex stress by $v_{1}$ is given by, $\int_{v_{1}}\left(v_{i}\right)=n-i$. Therefore, the total vertex stress induced by $v_{1}$ equals, $\mathfrak{s}_{P_{n}}\left(v_{1}\right)=\sum_{v_{j} \in V\left(P_{n}\right), j \neq 1} \int_{v_{1}}\left(v_{j}\right)=\frac{(n-1)(n-2)}{2}$. We now generalize this notion for a graph $G$.

Definition 1.1. Let $V(G)=\left\{v_{i}: 1 \leq i \leq n\right\}$ and for the ordered vertex pair $\left(v_{i}, v_{j}\right)$ let there be $k_{G}(i, j)$ distinct shortest paths of length $l_{G}(i, j)$ from $v_{i}$ to $v_{j}$. Then, $\mathfrak{s}_{G}\left(v_{i}\right)=\sum_{j=1, j \neq i}^{n} k_{G}(i, j)\left(\ell_{G}(i, j)-1\right)$.

It naturally follows that the total induced vertex stress of graph $G$ is $\mathfrak{s}(G)=$ $\sum_{i=1}^{n} \mathfrak{s}_{G}\left(v_{i}\right)$. Since we account for the shortest paths between an ordered vertex pair it follows that $\mathfrak{s}(G)$ is even. Therefore, an even number of vertices $v_{i}$ may exist for which $\mathfrak{s}_{G}\left(v_{i}\right)$ is an odd value. The aforesaid reminds of the classical result with regards to the number of vertices with odd degree. We also have a useful result which links this notion to the parameter of total vertex stress in the graph $G$.

Theorem 1.2. For a graph $G$ of order $n \geq 1$ we have $\mathfrak{s}(G)=2 \mathcal{S}(G)$.

Proof. Definition 1.1 implies that for a specific shortest $P=\left(v_{i}, v_{j}\right)$-path the sum

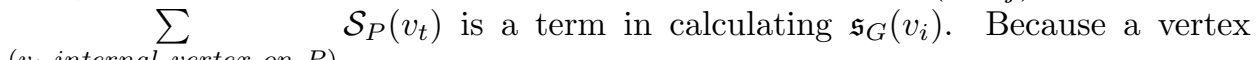
pair $\left(v_{i}, v_{j}\right)$ is an ordered pair, it follows that for the specific inverse $\left(v_{j}, v_{i}\right)$-path, the same term is recounted. Hence, throughout the graph $G$, each value $\mathcal{S}_{G}\left(v_{i}\right)$, $i=1,2,3, \ldots, n$ is counted twice. Therefore, the result $\mathfrak{s}(G)=2 \mathcal{S}(G)$.

Theorem 1.2 implies that for a stress regular graph [5], $\mathfrak{s}_{G}\left(v_{i}\right)=2 \mathcal{S}_{G}\left(v_{i}\right)$, $v_{i} \in V(G)$. We are now ready to move to the main sections of this paper.

The study focuses on the addition of an edge between two graphs as well as the merging of two vertices. Though the graph operations seem trivial, they reveal a complicated change in the total induced vertex stress. An important analytical application is found amongst others, in the field of mathematical chemistry. Many compound chemical structures are simply the addition of an edge between pairwise principle molecules (or nuclei) or chained molecules.

\section{Total INDUCED VERTEX STRESS OF TYPE 1 BARBELL-LIKE GRAPHS}

Recall that a barbell graph is a graph obtained by taking two copies of $C_{n}$, $n \geq 3$ and joining them with an edge. This idea can be generalized by joining any two graphs $G$ and $H$ with an edge. This family of graphs (infinitely many) is called the Type 1 barbell-like graphs. By convention, if $u_{i} \in V(G)$ and $v_{j} \in V(H)$ then joining the graphs with edge $u_{i} v_{j}$ will be denoted by $G \rightsquigarrow H\left(u_{i}, v_{j}\right)$ or equivalently by $H \rightsquigarrow G\left(v_{j}, u_{i}\right)$. 
Theorem 2.1. For graphs $G$ and $H$ of order $n$ and $m$ respectively, and $u_{i} \in V(G)$, $v_{j} \in V(H)$ and $k_{G}(t, i)$ the number of distinct shortest $\left(u_{t}, u_{i}\right)$-paths from $u_{t}$ to $u_{i}$ in $G$ and similarly, $k_{H}(t, j)$ the number of distinct shortest $\left(v_{t}, v_{j}\right)$-paths in $H$ it follows that,

$$
\begin{aligned}
\mathfrak{s}\left(G \rightsquigarrow H\left(u_{i}, v_{j}\right)\right) & =\mathfrak{s}(G)+\mathfrak{s}(H)+2\left[\mathfrak{s}_{G}\left(u_{i}\right)+\sum_{t=1, t \neq i}^{n} k_{G}(t, i)\right] \cdot\left[\sum_{s=1, s \neq j}^{m} k_{H}(s, j)+1\right] \\
& +2\left[\mathfrak{s}_{H}\left(v_{j}\right)+\sum_{s=1, s \neq j}^{m} k_{H}(s, j)\right] \cdot\left[\sum_{t=1, t \neq i}^{n} k_{G}(t, i)+1\right] .
\end{aligned}
$$

Proof. For the purpose of this proof we first prove the one-way partial result i.e. the total induced vertex stress from $V(G)$ to $V(H)$. We say that vertex $u_{t}$ induces vertex stress on transiting to vertex $v_{s}$ along all the shortest $\left(u_{t}, v_{s}\right)$-paths, $t=1,2,3, \ldots, n$ and $s=1,2,3, \ldots, m$.

(a) For the ordered vertex pairs $\left(u_{i}, v_{s}\right), s=1,2,3, \ldots, m$ the vertex $u_{i}$ induces total vertex stress of $\sum_{s=1, s \neq j}^{m} k_{H}(s, j)+\mathfrak{s}_{H}\left(v_{j}\right)$.

(b) Consider any specific vertex $u_{t} \in V(G) \backslash u_{i}$. For the ordered vertex pairs $\left(u_{t}, v_{s}\right)$, $s=1,2,3, \ldots, m$ the vertex $u_{t}$ induces a total of vertex stress of $k_{G}(t, i) \cdot\left[\left(\ell_{G}(t, i)\right.\right.$. $\left.\sum_{s=1, s \neq j}^{m} k_{H}(s, j)+\sum_{s=1, s \neq j}^{m} k_{H}(s, j)+\mathfrak{s}_{H}\left(v_{j}\right)\right]+k_{G}(t, i) \ell_{G}(t, i)$. Hence, $\forall u_{t} \in V(G) \backslash u_{i}$ the total induced vertex stress is given by $\sum_{t=1, t \neq i}^{n} k_{G}(t, i) \ell_{G}(t, i) \cdot\left[\sum_{s=1, s \neq j}^{m} k_{H}(s, j)+\right.$ $1]+\sum_{t=1, t \neq i}^{n} k_{G}(t, i)\left[\sum_{s=1, s \neq j}^{m} k_{H}(s, j)+\mathfrak{s}_{H}\left(v_{j}\right)\right]$. Thus, the one-way partial result i.e. the total induced vertex stress from $V(G)$ to $V(H)$ is given by,

$\sum_{s=1, s \neq j}^{m} k_{H}(s, j)+\mathfrak{s}_{H}\left(v_{j}\right)+\left[\mathfrak{s}_{G}\left(u_{i}\right)+\sum_{t=1, t \neq i}^{n} k_{G}(t, i)\right] \cdot\left[1+\sum_{s=1, s \neq j}^{m} k_{H}(s, j)\right]+\sum_{t=1, t \neq i}^{n} k_{G}(t, i)$. $\left[\sum_{s=1, s \neq j}^{m} k_{H}(s, j)+\mathfrak{s}_{H}\left(v_{j}\right)\right]$. For all inverse $\left(v_{j}, u_{i}\right)$-paths the total vertex stress induced by $V(H)$ to $V(G)$ is given by,

$$
\begin{aligned}
& \sum_{t=1, t \neq i}^{n} k_{G}(t, i)+\mathfrak{s}_{G}\left(u_{i}\right)+\left[\mathfrak{s}_{H}\left(v_{j}\right)+\sum_{s=1, s \neq j}^{m} k_{H}(s, j)\right] \cdot\left[1+\sum_{t=1, t \neq i}^{n} k_{G}(t, i)\right]+\sum_{s=1, s \neq j}^{m} k_{H}(s, j) . \\
& {\left[\sum_{\substack{t=1, t \neq i \\
\text { with. Adding all parts and simplifying yield the result. }}}^{n} k_{G}(t, i)+\mathfrak{s}_{G}\left(u_{i}\right)\right] . \text { In addition we have the values } \mathfrak{s}(G) \text { and } \mathfrak{s}(H) \text { to begin }}
\end{aligned}
$$

A more complex graph can now be addressed. Consider the graphs $H_{i}, 1 \leq$ $i \leq \ell$ and let $G=\left(\left(\left(\left(H_{1} \rightsquigarrow H_{2}\left(u_{i}, v_{j}\right)\right) \rightsquigarrow H_{3}\left(v_{t}, w_{s}\right)\right) \rightsquigarrow \cdots\right) \rightsquigarrow H_{\ell}\left(y_{q}, z_{r}\right)\right)$. Put differently, for graphs $H_{i}, i=1,2,3, \ldots, \ell$ and $G_{1}=H_{1} \rightsquigarrow H_{2}(u, v), u \in V\left(H_{1}\right)$, $v \in V\left(H_{2}\right)$ let, $G_{j}=G_{j-1} \rightsquigarrow H_{j+1}(u, v), u \in V\left(G_{j-1}\right), v \in V\left(H_{j+1}\right)$ and $2 \leq$ $j \leq \ell-1$. The result from Theorem 2.1 can be applied to recursively determine the total induced vertex stress. However, with each recursive step the vertex stress 
induced by each vertex must be updated accordingly. The next corollary from the proof of Theorem 2.1, provides these new values.

Corollary 2.2. In the graph $Q=G \rightsquigarrow H\left(u_{i}, v_{j}\right)$ we have:

(a) $\mathfrak{s}_{Q}\left(u_{i}\right)=\mathfrak{s}_{G}\left(u_{i}\right)+\mathfrak{s}_{H}\left(v_{j}\right)+\sum_{s=1, s \neq j}^{m} k_{H}(s, j)$.

(b) $\mathfrak{s}_{Q}\left(u_{t}\right)_{t \neq i}=\mathfrak{s}_{G}\left(u_{t}\right)+k_{G}(t, i) \ell_{G}(t, i)\left[1+\sum_{s=1, s \neq j}^{m} k_{H}(s, j)\right]+k_{G}(t, i)\left[\mathfrak{s}_{H}\left(v_{j}\right)+\right.$

$\left.\sum_{s=1, s \neq j}^{m} k_{H}(s, j)\right], t=1,2,3, \ldots, n, t \neq i$.

(c) $\mathfrak{s}_{Q}\left(v_{j}\right)=\mathfrak{s}_{H}\left(v_{j}\right)+\mathfrak{s}_{G}\left(u_{i}\right)+\sum_{t=1, t \neq i}^{n} k_{G}(t, i)$.

(d) $\mathfrak{s}_{Q}\left(v_{s}\right)_{s \neq j}=\mathfrak{s}_{H}\left(v_{s}\right)+k_{H}(s, j) \ell_{H}(s, j)\left[1+\sum_{t=1, t \neq i}^{n} k_{G}(t, i)\right]+k_{H}(s, j)\left[\mathfrak{s}_{G}\left(u_{i}\right)+\right.$ $\left.\sum_{t=1, t \neq i}^{n} k_{G}(t, i)\right], s=1,2,3, \ldots, m, s \neq j$.

2.1. Maximum and minimum total induced vertex stress. Note that in the result of Theorem 2.1, the terms $\mathfrak{s}(G)$ and $\mathfrak{s}(H)$ are constants whilst all other terms may vary by the choice of $u_{i}, v_{j}$. This observation suggests that over all such distinct pairwise choices, the parameter $\mathfrak{s}\left(G \rightsquigarrow H\left(u_{i}, v_{j}\right)\right)$ will attain a maximum and a minimum value.

Theorem 2.3. Let graph $G$ be of order $n \geq 1$. If $\operatorname{deg}_{G}\left(u_{i}\right) \leq \operatorname{deg}_{G}\left(u_{j}\right)$ then $\mathfrak{s}_{G}\left(u_{i}\right) \geq \mathfrak{s}_{G}\left(u_{j}\right)$.

Proof. For $n=1,2,3,4$ and up to isomorphism, there are only 1, 1, 2 and 6 connected simple graphs respectively. It is easy to verify that the result holds for order $1 \leq n \leq 4$. Now consider any connected simple graph of order $n \geq 5$. Since, any such graph $G$ is a subgraph of $K_{n}$ we may begin with $K_{n}$ and through iterative deletion of an edge at a time, reconstruct $G$. For $K_{n}$ the result holds since $\operatorname{deg}_{K_{n}}\left(v_{i}\right)=\delta\left(K_{n}\right), \forall v_{i} \in V\left(K_{n}\right)$.

Without loss of generality let $V(G)=\left\{u_{i}: 1 \leq i \leq n\right\}$ such that $\operatorname{deg}_{G}\left(u_{1}\right) \leq$ $\operatorname{deg}_{G}\left(u_{2}\right) \leq \cdots \leq \operatorname{deg}_{G}\left(u_{n}\right)$. Pair the vertices $u_{i}$ and $v_{i}$ in a one-on-one association. The vertices $v_{j}$ which associate with the closed neighborhood $N\left[u_{i}\right]$ will be denoted by $N_{G}\left[v_{i}\right]$. By convention $\iota\left(v_{j}\right)=j$. Begin with $v_{1}$ and from $V\left(K_{n}\right) \backslash N\left[v_{1}\right]$ select the smallest $\iota\left(v_{k_{1}}\right)$ and delete edge $v_{1} v_{k_{1}}$. Clearly, vertices $v_{1}, v_{k_{1}}$ each induce a total of vertex stress equal to $n-2$. Hence, for $Q_{1,1}=K_{n}-v_{1} v_{k_{1}}$ we have $\mathfrak{s}_{Q_{1,1}}\left(v_{1}\right)=\mathfrak{s}_{Q_{1,1}}\left(v_{k_{1}}\right)=n-2$ and $\mathfrak{s}_{Q_{1,1}}\left(v_{t}\right)=0, \forall v_{t}, t \neq 1, k_{1}$. Thus the result holds for $K_{n}-v_{1} v_{k_{1}}$. Now consider $\left(V\left(K_{n}\right) \backslash N\left[v_{1}\right]\right) \backslash v_{k_{1}}$ and select the smallest $\iota\left(v_{k_{2}}\right)$. Delete the edge $v_{1} v_{k_{2}}$. Clearly in $Q_{1,2}=K_{n}-\left\{v_{1} v_{k_{1}}, v_{1} v_{k_{2}}\right\}$ the induced vertex stress is given by $\mathfrak{s}_{Q_{1,2}}\left(v_{1}\right)=2(n-3), \mathfrak{s}_{Q_{1,2}}\left(v_{k_{1}}\right)=(n-3)$, $\mathfrak{s}_{Q_{1,2}}\left(v_{k_{2}}\right)=(n-3)$ and $\mathfrak{s}_{Q_{1,2}}\left(v_{t}\right)=0, \forall v_{t}, t \neq 1, k_{1}, k_{2}$. Therefore result holds for $K_{n}-\left\{v_{1} v_{k_{1}}, v_{1} v_{k_{2}}\right\}$. Repeat the procedure until $\operatorname{deg}_{Q_{1, r}}\left(v_{1}\right)=\operatorname{deg}_{G}\left(u_{1}\right)$. Clearly the results holds.

Consider $v_{2}$. If $\operatorname{deg}_{Q_{1, r}}\left(v_{2}\right)=\operatorname{deg}_{G}\left(u_{2}\right)$ the results holds. 
If $\operatorname{deg}_{Q_{1}, r}\left(v_{2}\right)>\operatorname{deg}_{G}\left(u_{2}\right)$, follow the described procedure to delete edges. When $\operatorname{deg}_{Q_{2, q}}\left(v_{2}\right)=\operatorname{deg}_{G}\left(u_{2}\right)$ is reached, then two possibilities exist. Firstly, if $\operatorname{deg}_{G}\left(u_{1}\right)<$ $\operatorname{deg}_{G}\left(u_{2}\right)$, then $\mathfrak{s}_{Q_{2, q}}\left(v_{1}\right) \geq \mathfrak{s}_{Q_{2, q}}\left(v_{2}\right)$. Secondly, if $\operatorname{deg}_{G}\left(u_{1}\right)=\operatorname{deg}_{G}\left(u_{2}\right)$ then $\mathfrak{s}_{Q_{2, q}}\left(v_{1}\right) \leq \mathfrak{s}_{Q_{2, q}}\left(v_{2}\right)$. However, for both cases the result holds i.e. for $v_{1}$ or $v_{2}$ since both, $\operatorname{deg}_{G}\left(u_{1}\right)=\operatorname{deg}_{G}\left(u_{2}\right)=\delta(G)$. Through immediate induction, the result holds for $G$ of order $n$. Since $G$ and $n$ were random, it follows by imbedded induction that the result i.e. if $\operatorname{deg}_{G}\left(u_{i}\right) \leq \operatorname{deg}_{G}\left(u_{j}\right)$ then $\mathfrak{s}_{G}\left(u_{i}\right) \geq \mathfrak{s}_{G}\left(u_{j}\right)$ holds for all graphs $G$ of order $n \geq 1$.

For the application of Theorem 2.3, we note specific interpretations. For a graph $G$ of order $n \geq 1$ it follows that:

(a) There exists a vertex $u_{i} \in V(G)$ such that $\operatorname{deg}_{G}\left(u_{i}\right)=\delta(G)$ and $\mathfrak{s}_{G}\left(u_{i}\right)=$ $\max \left\{\mathfrak{s}_{G}\left(u_{j}\right): u_{j} \in V(G)\right\}$.

(b) There exists a vertex $u_{i} \in V(G)$ such that $\operatorname{deg}_{G}\left(u_{i}\right)=\Delta(G)$ and $\mathfrak{s}_{G}\left(u_{i}\right)=$ $\min \left\{\mathfrak{s}_{G}\left(u_{j}\right): u_{j} \in V(G)\right\}$.

Note that (b) is a corollary from Theorem 2.3 which the reader may prove formally. Consider the sets $X=\left\{u_{i}: \mathfrak{s}_{G}\left(u_{i}\right)\right.$ is a maximum in $\left.G\right\}$ and $Y=\left\{u_{j}: \mathfrak{s}_{G}\left(u_{j}\right)\right.$ is a minimum in $G$ \}.

Lemma 2.4. For a graph $G$ of order $n \geq 1$ let $k_{G}(i, j)$ be the number of distinct shortest $\left(u_{i}, u_{j}\right)$-paths, $1 \leq j_{\neq i} \leq n$ of length $\ell_{G}(i, j)$. It follows that:

(a) There exists a vertex $u_{i} \in X$ such that $\mathfrak{s}_{G}\left(u_{i}\right)+\sum_{j=1, j \neq i}^{n} \ell_{G}(i, j)$ is a maximum.

(b) There exists a vertex $u_{i} \in Y$ such that $\mathfrak{s}_{G}\left(u_{i}\right)+\sum_{j=1, j \neq i}^{n} \ell_{G}(i, j)$ is a minimum.

Proof. Both (a) and (b) follow from the fact that $k_{G}(i, j)$ is well-defined for all $u_{i} \in V(G)$ and Theorem 2.3 yields the other required term.

Theorem 2.5. (a) For graphs $G$ and $H$ of order $n$ and $m$ respectively, let $u_{i} \in$ $V(G)$ be such that $\mathfrak{s}_{G}\left(u_{i}\right)+\sum_{j=1, j \neq i}^{n} \ell_{G}(i, j)$ is a maximum and let $v_{j} \in V(H)$ be such that $\mathfrak{s}_{H}\left(v_{j}\right)+\sum_{i=1, i \neq j}^{m} \ell_{G}(j, i)$ is a maximum. Then $\mathfrak{s}\left(G \rightsquigarrow H\left(u_{i}, v_{j}\right)\right)$ is a maximum. (b) For graphs $G$ and $H$ of order $n$ and $m$ respectively, let $u_{i} \in V(G)$ be such that $\mathfrak{s}_{G}\left(u_{i}\right)+\sum_{j=1, j \neq i}^{n} \ell_{G}(i, j)$ is a minimum and let $v_{j} \in V(H)$ be such that $\mathfrak{s}_{H}\left(v_{j}\right)+$ $\sum_{i=1, i \neq j}^{m} \ell_{G}(j, i)$ is a minimum. Then $\mathfrak{s}\left(G \rightsquigarrow H\left(u_{i}, v_{j}\right)\right)$ is a minimum.

Proof. The result is a direct consequence of Theorem 2.3 and Lemma 2.4. 


\section{TOtAl INDUCED VERTEX STRESS OF TYPE 2 BARBELL-LIKE GRAPHS}

Joining any two graphs $G$ and $H$ by merging vertex $u_{i} \in V(G)$ with vertex $v_{j} \in V(H)$ results in a Type 2 barbell-like graph. By convention, such new graph is denoted by, $G \odot H\left(u_{i}, v_{j}\right)$ or equivalently by $H \odot G\left(v_{j}, u_{i}\right)$.

Theorem 3.1. For graphs $G$ and $H$ of order $n$ and $m$ respectively, and $u_{i} \in V(G)$, $v_{j} \in V(H)$ and $k_{G}(t, i)$ the number of distinct shortest $\left(u_{t}, u_{i}\right)$-paths from $u_{t}$ to $u_{i}$ in $G$ and similarly, $k_{H}(t, j)$ the number of distinct shortest $\left(v_{t}, v_{j}\right)$-paths in $H$ it follows that,

$$
\begin{gathered}
\mathfrak{s}\left(G \odot H\left(u_{i}, v_{j}\right)\right)=\mathfrak{s}(G)+\mathfrak{s}(H)+2\left[\mathfrak{s}_{G}\left(u_{i}\right)+\sum_{t=1, t \neq i}^{n} k_{G}(t, i)\right] \cdot \sum_{t=1, t \neq j}^{m} k_{H}(t, j)+ \\
2 \sum_{t=1, t \neq i}^{n} k_{G}(t, i) \cdot \mathfrak{s}_{H}\left(v_{j}\right) .
\end{gathered}
$$

Proof. Suffice to say that the result follows from the result of Theorem 2.1 by contracting the edge $u_{i} v_{j}$.

A more complex graph can now be addressed. Consider the graphs $H_{i}, 1 \leq$ $i \leq \ell$ and let $G=\left(\left(\left(\left(H_{1} \odot H_{2}\left(u_{i}, v_{j}\right)\right) \odot H_{3}\left(v_{t}, w_{s}\right)\right) \odot \cdots\right) \odot H_{\ell}\left(y_{q}, z_{r}\right)\right)$. Put differently, for graphs $H_{i}, i=1,2,3, \ldots, \ell$ and $G_{1}=H_{1} \odot H_{2}(u, v), u \in V\left(H_{1}\right)$, $v \in V\left(H_{2}\right)$ let, $G_{j}=G_{j-1} \odot H_{j+1}(u, v), u \in V\left(G_{j-1}\right), v \in V\left(H_{j+1}\right)$ and $2 \leq$ $j \leq \ell-1$. The result from Theorem 3.1 can be applied to recursively determine the total induced vertex stress. However, with each recursive step the vertex stress induced by each vertex must be updated accordingly. The next corollary from the proof of Theorem 3.1 provides these new values.

Corollary 3.2. In the graph $Q=G \odot H\left(u_{i}, v_{j}\right)$ we have:

(a) $\mathfrak{s}_{Q}\left(u_{i}\right)=\mathfrak{s}_{G}\left(u_{i}\right)+\mathfrak{s}_{H}\left(v_{j}\right)$.

(b) $\mathfrak{s}_{Q}\left(u_{t}\right)_{t \neq i}=\mathfrak{s}_{G}\left(u_{t}\right)+k_{G}(t, i) \ell_{G}(t, i) \sum_{s=1, s \neq j}^{m} k_{H}(s, j)+\mathfrak{s}_{H}\left(v_{j}\right) k_{G}(t, i), t=1,2,3, \ldots, n$, $t \neq i$.

(c) $\mathfrak{s}_{Q}\left(v_{j}\right)=\mathfrak{s}_{H}\left(v_{j}\right)+\mathfrak{s}_{G}\left(u_{i}\right)$.

(d) $\mathfrak{s}_{Q}\left(v_{s}\right)_{s \neq j}=\mathfrak{s}_{H}\left(v_{s}\right)+k_{H}(s, j) \ell_{H}(s, j) \sum_{t=1, t \neq i}^{n} k_{G}(t, i)+\mathfrak{s}_{G}\left(u_{i}\right) k_{H}(s, j), \quad s=$ $1,2,3, \ldots, m, s \neq j$.

3.1. Maximum and minimum total induced vertex stress. Following Theorem 2.3 and the interpretations thereof as well as utilizing Lemma 2.4 we state the next theorem without proof.

Theorem 3.3. (a) For graphs $G$ and $H$ of order $n$ and $m$ respectively, let $u_{i} \in$ $V(G)$ be such that $\mathfrak{s}_{G}\left(u_{i}\right)+\sum_{j=1, j \neq i}^{n} \ell_{G}(i, j)$ is a maximum and let $v_{j} \in V(H)$ be such that $\mathfrak{s}_{H}\left(v_{j}\right)+\sum_{i=1, i \neq j}^{m} \ell_{G}(j, i)$ is a maximum. Then $\mathfrak{s}\left(G \odot H\left(u_{i}, v_{j}\right)\right)$ is a maximum. 
(b) For graphs $G$ and $H$ of order $n$ and $m$ respectively, let $u_{i} \in V(G)$ be such that $\mathfrak{s}_{G}\left(u_{i}\right)+\sum_{j=1, j \neq i}^{n} \ell_{G}(i, j)$ is a minimum and let $v_{j} \in V(H)$ be such that $\mathfrak{s}_{H}\left(v_{j}\right)+$ $\sum_{i=1, i \neq j}^{m} \ell_{G}(j, i)$ is a minimum. Then $\mathfrak{s}\left(G \odot H\left(u_{i}, v_{j}\right)\right)$ is a minimum.

\section{Conclusion}

Consider the complete graph $K_{n}, n \geq 2$ as well as graphs $G_{j}, j=1,2,3, \ldots, n$. Merge any vertex $u_{i} \in V\left(G_{j}\right)$ with vertex $v_{j} \in K_{n}$. Hence, $K_{n}$ is a cut-clique in the compound graph $H_{1}$ we constructed. If all edges of $K_{n}$ are contracted a graph $\mathrm{H}_{2}$ is obtained which contains a compound cut vertex.

Problem 1. If possible, find an elegant way of calculating $\mathfrak{s}\left(H_{1}\right)$ and $\mathfrak{s}\left(H_{2}\right)$ through the recursive utilization of Theorems 2.1 and 3.1 .

Problem 2. Design and code an efficient algorithm which integrates the results of Theorems 2.1, 2.3, Corollary 2.2 and Lemma 2.4 to facilitate research into complex graphs.

Conventionally, if graphs $G$ of order $n$ and $H$ are disconnected then distance $d(u, v)=\infty$ for $u \in V(G), v \in V(H)$. For the study of either vertex stress or induced vertex stress we define $d(u, v)=0$ for $u \in V(G), v \in V(H)$. It implies that for the disjoint union, $\mathfrak{s}(G \cup H)=\mathfrak{s}(G)+\mathfrak{s}(H)$. In the join $G+H$ all distances between the vertices of $V(G) \cup V(H)$ reduce to either 1 or 2 . In the corona $G \circ H$ (hence, $n$ copies of $H$ ) all distances in each copy of $H$ reduce to either 1 or 2 .

Problem 3. Study total vertex stress and total induced vertex stress in $G+H$ and $G \circ H$.

Problem 4. Study total vertex stress and total induced vertex stress in $G \mathfrak{p} H$, with $\mathfrak{p}$ other graph products.

Theorem 1.2 implies that for self-complementary graphs $G, \mathcal{S}(G)+\mathcal{S}(\bar{G})=\mathfrak{s}(G)$. Thus, in general $\mathcal{S}(G)+\mathcal{S}(\bar{G}) \leq \max \{\mathfrak{s}(G), \mathfrak{s}(\bar{G})\}$.

Problem 5. Investigate the existence, if any, of Nordhaus-Gaddum relations in respect of total vertex stress.

The study of vertex stress (atom stress) and total vertex stress in a graph (molecular stress) and the notion of induced vertex stress (induced atomic stress) in chemical structures renders wide scope for further research. It is hoped that such research will contribute to a deeper understanding of chemical properties and contribute to the field of mathematical chemistry.

Acknowledgement. The authors express their appreciation for the constructive comments and guidance by the anonymous referees.

\section{REFERENCES}

[1] Bondy, J.A. and Murty, U.S.R., Graph Theory with Applications, Macmillan Press, London, 1976.

[2] Harary, F., Graph Theory, Addison-Wesley, Reading MA, 1969. 
[3] Kok, J., Shiny, J. and Ajitha, V., "Total vertex stress alteration in cycle graphs", Communicated.

[4] Shimbel, A., "Structural parameters of communication networks", The Bulletin of Mathematical Biophysics, 15 no. 4, (1953), 501-507.

[5] Shiny, J. and Ajitha, V., "Stress regular graphs", Malaya Journal of Matematik, 8 no. 3, (2020), 1152-1154.

[6] West, B., Introduction to Graph Theory, Prentice-Hall, Upper Saddle River, 1996. 